\title{
Propagation conditions of an eccentric crack in a semi-circular disk loaded in I+II mixed mode
}

\author{
Lucie Malíková ${ }^{1,2, *}$, Petr Miarka ${ }^{1,2}$, and Hana Šimonová ${ }^{1}$ \\ ${ }^{1}$ Brno University of Technology, Faculty of Civil Engineering, Institute of Structural Mechanics, \\ Veveř́ 331/95, 60200 Brno, Czech Republic \\ ${ }^{2}$ Institute of Physics of Materials, Czech Academy of Sciences, v. v. i., Žižkova 513/22, 61662 Brno, \\ Czech Republic
}

\begin{abstract}
Multi-parameter fracture mechanics concept is applied to investigate crack propagation conditions during a three-point bending (3PB) test performed on an asymmetrical semi-circular disk under I+II mixedmode loading. The asymmetry is represented by an eccentricity of the angled crack as well as by different distances of both supports during the 3PB test from the middle of the specimen. The Williams series expansion is used for the crack-tip stress field approximation and fracture response of the crack is assessed by means of the combination of the numerical and analytical solution. Comparison between the individual configurations is carried out and discussion on the importance of the higher-order terms considered in the Williams expansion for various cases of asymmetry is presented.
\end{abstract}

\section{Introduction}

Fracture mechanics, that is necessary for assessment of fracture response of structures made of different materials, develops permanently. In the engineering praxis, more and more advanced materials are used because of their tailored properties. Various requests can be made on such materials, such as combination of their apparently opposite properties, or just a utilization of more environment-friendly materials. Anyway, new approaches of fracture mechanics concepts need to be derived in order to be able to describe the fracture behaviour of materials with non-brittle manner of damage.

One of the recent efforts is more accurate approximation of the crack-tip stress and/or displacement fields that is crucial for additional more comprehensive fracture analyses. The multi-parameter/generalized approach proved to be effective in that case $[1,2,3]$. This concept is necessary when the fracture processes occur in a more distant surrounding from the crack tip, where the classical singular single-parameter solution is no more valid. Then, the Williams expansion [4] can be utilized assuming several initial terms of the power series. For example, in $[5,6]$ an advanced form of the common maximum tangential stress criterion for estimation of the further crack propagation is applied considering higher-order terms of the crack-tip stress field approximation. Smith et al. [6] predicted the fracture onset in PMMA specimens by assuming the first non-singular term ( $T$-stress) of the Williams expansion. The

\footnotetext{
* Corresponding author: malikova.1@ffe.vutbr.cz
} 
fracture predictions were shown to be more accurate taking into account the $T$-stress term, specially under mode II loading. Ayatollahi and Nejati [7, 8] computed the higher-order terms coefficients of for cracked as well as $\mathrm{V}$-notched specimens by using a numeral procedure called the over-deterministic method (ODM), that is also applied within this work. They also proved that the non-singular terms of the series play an important role in the derivation of crack-tip stress approximation. Several recent works of the authors' collective are devoted to the investigation of the significance of the truncated form of the power expansion as well $[9,10,11,12]$.

In this work, a numerical analysis is performed on cracked semi-circular discs under bending (SCB). The inclined crack causes its loading under I + II mixed mode and its initial propagation angle is investigated by means of generalized MTS criterion considering nonsingular higher-order terms. Moreover, two kinds of eccentricities are simulated - eccentric crack as well as eccentric supports during the three-point bending test. Configurations with the most influence of the higher-order terms on the crack propagation predictions are searched in order to suggest suitable configurations to be tested experimentally. A comparison between the numerical and experimental results is planned in the future work.

\section{Theoretical background}

The following points need to be introduced before the analysis with its results can be presented: Williams power expansion, over-deterministic method, fracture criterion.

\subsection{Williams power expansion}

Williams power expansion was derived in 1957 by Williams [4] as a solution of the cracktip stress/displacement field approximation. The Williams power series for stress/displacement distribution was originally derived for a homogeneous isotropic elastic cracked body subjected to an arbitrary remote loading:

$$
\begin{aligned}
& \sigma_{i j}=\sum_{n=1}^{\infty} \frac{n}{2} r^{\frac{n}{2}-1} f_{i j}(n, \theta) A_{n}+\sum_{m=1}^{\infty} \frac{m}{2} r^{\frac{m}{2}-1} g_{i j}(m, \theta) B_{m} \quad \text { where } i, j \in\{x, y\} \\
& u_{i}=\sum_{n=0}^{\infty} r^{\frac{n}{2}} f_{i}(n, \theta, E, v) A_{n}+\sum_{m=0}^{\infty} r^{\frac{m}{2}} g_{i}(m, \theta, E, v) B_{m} \text { where } i \in\{x, y\} .
\end{aligned}
$$

The meaning of the symbols used in Eq. 1 and 2 is following: $\sigma_{i j}$ and $u_{i}$ represents stress tensor and displacement vector component, respectively; $(r, \theta)$ stand for polar system coordinates; $A_{n}$ and $B_{m}$ are coefficients of the series expansion and must be determined numerically, see the next section; $E$ and $v$ denotes elastic properties of the material (Young's modulus and Poisson's ratio, respectively); relations for calculation of the functions $f$ and $g$ can be found in literature.

\subsection{Over-deterministic method}

When the multi-parameter form of the Williams expansion with higher-order terms shall be considered for the stress/displacement crack-tip fields approximation, it is necessary to determine the coefficients of the higher-order terms. This is performed numerically and so far, several methods have been derived and tested, for instance [13, 14, 15]. In this work, the over-deterministic method (ODM) suggested by Ayatollahi and Nejati [7] is utilized. More details on the procedure can be found also in [16]. The ODM is based on the solution of an over-determined system of equations: directly according to Eq. $2,2 k$ of equations for 
displacements $u_{x}$ and $u_{y}$ of $k$ nodes around the crack tip are formed and it has to hold that the total number of unknowns (coefficients of mode I and mode II higher-order terms) is lower than the number of equations, i.e. $N+M+2<2 k$.

\subsection{Fracture criterion}

When the crack-tip stress field approximation is prepared, a suitable criterion for estimation of the further crack propagation angle can be chosen. In this paper, the well-known maximum tangential stress (MTS) criterion is applied, originally derived by Erdogan and Sih [17]. Particularly, its multi-parameter/generalized form is utilized, i.e. the tangential stress component is expressed via the Williams expansion considering various numbers of the initial terms and the direction where $\sigma_{\theta \theta}$ becomes maximum represents the angle of the crack deflection. Note that the classical MTS criterion decides about the crack deflection angle exclusively from the values of the stress intensity factors as the only-controlling singular parameters. Considering also the non-singular terms of the Williams expansion causes arising of new a length parameter in the criterion that is often called as a critical distance and several theories can be found in literature about its suitable choice $[18,19,20]$.

\section{Semi-circular disc under 3-Point-Bending (3PB) test}

A I + II mixed mode configuration was selected to be able to assess the influence of the higher-order terms of the Williams expansion on the estimation of the crack deflection angle. Particularly, a semi-circular disc under three-point bending (SCB) with a skew crack has been investigated. Two kinds of eccentricities were modelled in a finite element software: crack eccentricity and supports asymmetry, see the schema in Fig. 1. The numerical analysis is essential in order to get the proper nodal solution for the ODM procedure enabling determination of the higher-order terms of the Williams expansion.

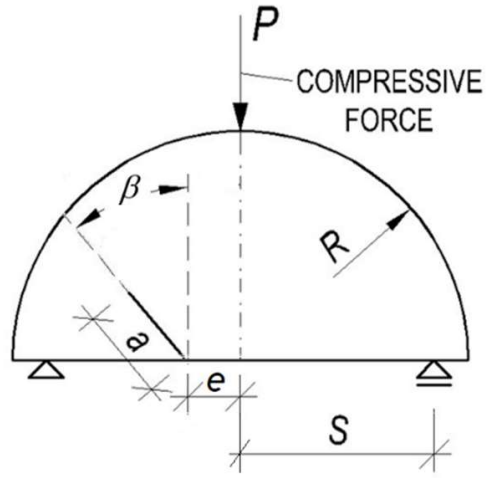

a) various crack eccentricity

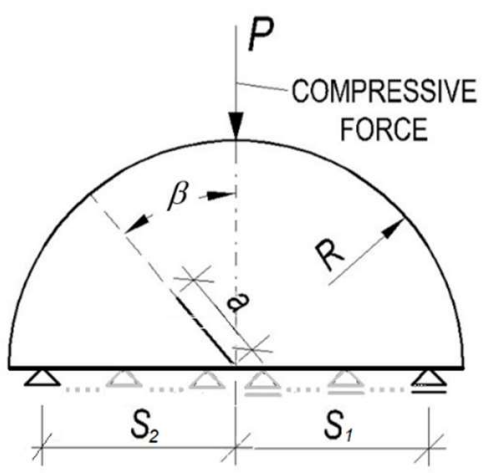

b) asymmetric supports

Fig. 1. Schema of the semi-circular specimen under three-point bending (SCB): (a) with various crack eccentricity; (b) with asymmetric supports.

Based on the previous analyses, several parameters were kept constant whereas other ones were varied to perform a parametric study. During the investigations, only the configurations with opening crack were considered; crack closure was not allowed. Thus, the following set of dimensions was applied to the numerical model:

- $\quad$ radius of the disc, $R=50 \mathrm{~mm}$; 
- $\quad$ crack length, $a=10$ and $25 \mathrm{~mm}$ (i.e. relative crack length $a / R=0.2$ and 0.5 );

- crack inclination angle, $\beta=30^{\circ} \div 50^{\circ}$ (with the step of $10^{\circ}$ ).

The previous parameters are valid for both kinds of studies, crack eccentricity as well as supports asymmetry. The rest of the parameters differs in this way:

(a) eccentric crack configurations

- $\quad$ relative half span between the supports, $S / R=0.8$;

- crack eccentricity, $e=-15,-10,-5,5,10$ and $15 \mathrm{~mm}$ from the center (note that positive values represents the eccentricity as depicted in Fig. 1 and negative values represents the crack shifted in the opposite way).

(b) asymmetric supports configurations

- distance of the supports from the center, $S_{I}=10,20,30$ and $40 \mathrm{~mm}, S_{2}=30$ and $40 \mathrm{~mm}$;

- crack eccentricity, $e=0 \mathrm{~mm}$ from the center.

Young's modulus of $35 \mathrm{GPa}$ and Poisson's ratio of 0.23 were set as elastic properties of the numerical model. This corresponds to the mechanical properties of a novel fine-grained composite based on alkali-activated matrix whose fracture response shall be tested under mixed mode.

More details on the numerical model parameters set in the commercial finite element software ANSYS [21] can be summarized via following items:

- the geometry of the numerical model was created through the quadrilateral element PLANE183;

- refinement of the mesh at the crack tip was performed;

- special crack elements with shifted mid-side nodes were located directly at the crack tip to support the square-root stress singularity;

- plane strain boundary conditions were prescribed;

- a compressive force $P=1 \mathrm{kN}$ was applied on the top of the SCB specimen;

- a ring of nodes at the distance of $4 \mathrm{~mm}$ from the crack tip was used for evaluation of the ODM procedure;

- multi-parameter MTS criterion was assessed at the critical distances $r_{c}=0.1,0.5$ and $1 \mathrm{~mm}$ assuming from 1 to 10 initial terms of the Williams power series for expression of the tangential stress.

\section{Results}

Because of the large extent of the results corresponding to many various configurations, only selected dependences of the crack deflection angle are presented.

The former analysis was devoted to investigations on SCB specimens with the crack shifted from the middle of the specimen (eccentric crack configurations, see Fig. 1a). Various crack eccentricities $e$ in combination with various initial crack inclination angles $\beta$ lead to different mixed-mode conditions that are expressed via the stress intensity factors ratio $K_{\mathrm{I}} / K_{\mathrm{II}}$. Results obtained for a crack with its relative length $a / R=0.2$ are summarized in Fig. 2; individual plots (a-c) correspond to different critical distances $r_{c}$, where the multi-parameter MTS criterion was applied and curves for different numbers of the initial terms considered in Williams expansion for $\sigma_{\theta \theta}$ approximation are plotted in different colors and symbols. 


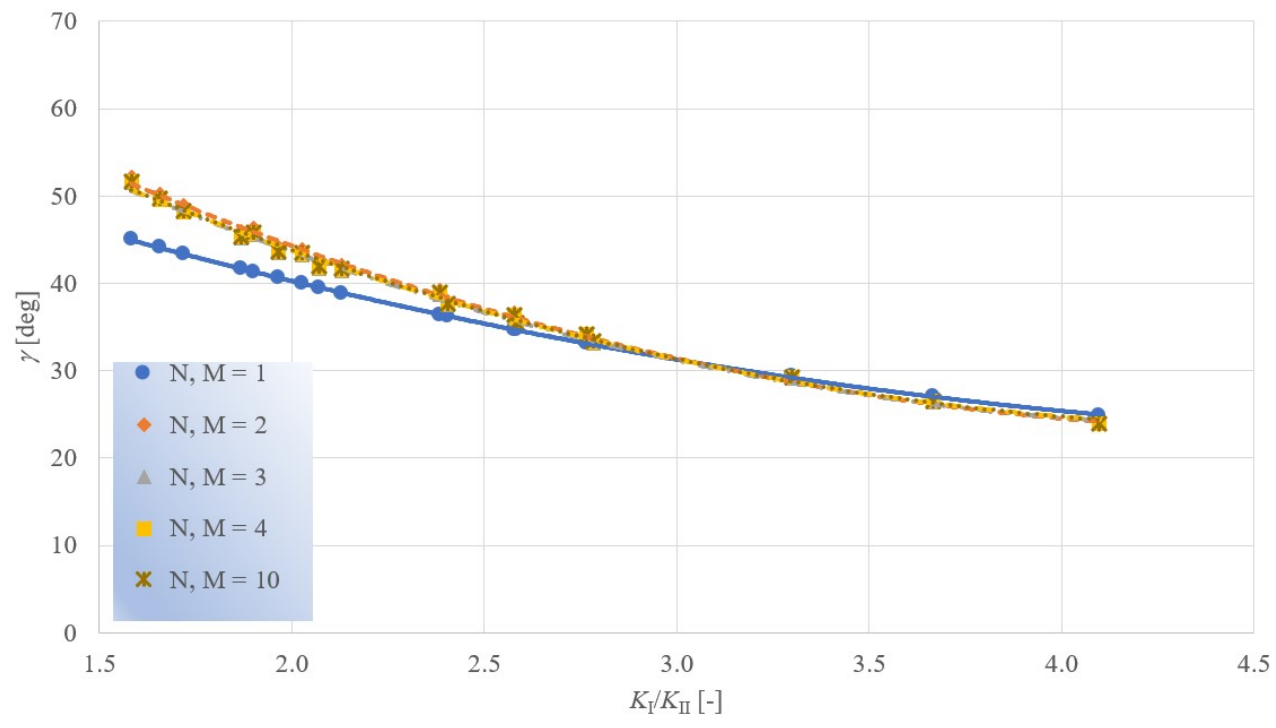

a) $r_{c}=0.1 \mathrm{~mm}$

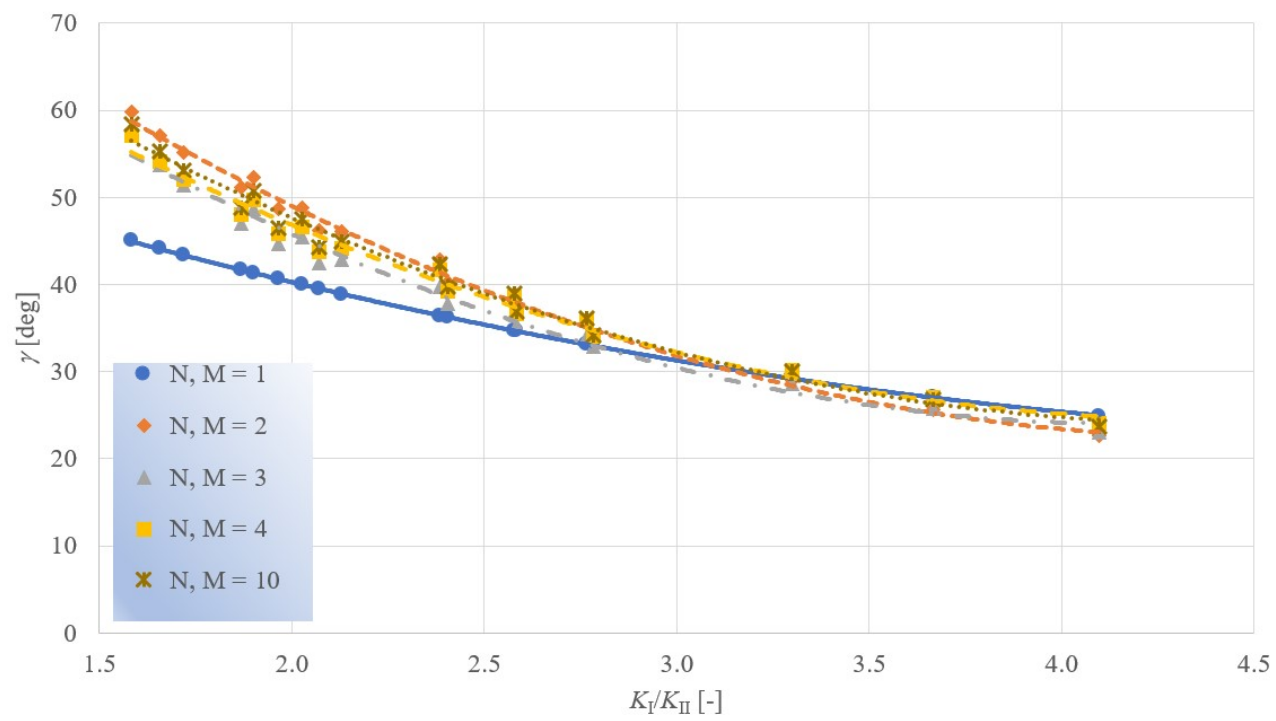

b) $r_{c}=0.5 \mathrm{~mm}$ 


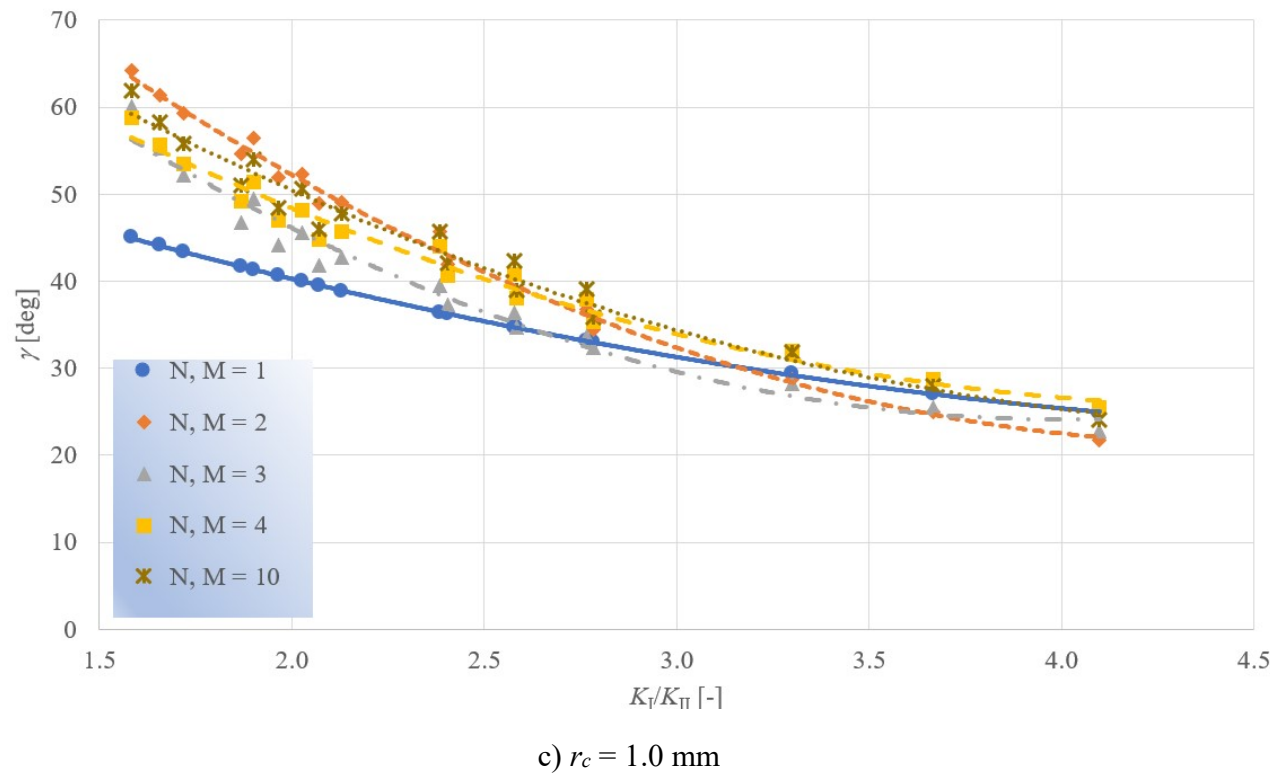

Fig. 2. Dependences of the crack deflection angle $\gamma$ on the $K_{\mathrm{I}} / K_{\mathrm{II}}$ ratio obtained on SCB specimens with various crack eccentricities $(a / R=0.2)$ at different critical distances $r_{c} ; N$ and $M$ represents the numbers of the initial terms considered in Williams expansion for $\sigma \theta \theta$ approximation.

The basic conclusion that can be seen from Fig. 2 is that the classical (only stress intensity factors based) MTS criterion gives quite different values of the crack deflection angle than the multi-parameter one for some specific configurations. It is therefore reasonable consider more than the first singular term for the crack-tip stress field description.

The plots in Fig. 2 show that when the stress intensity factors ratio decreases towards the value of 1, the importance of the higher-order terms of the Williams expansion increases. This is the case of positive crack eccentricities $e$ (see Fig. 1a) when the shear loading mode becomes significant and comparable to the opening one. This effect is more obvious and relevant when larger distances are used for fracture criterion application, i.e. for materials where the fracture processes occur in larger region around the crack tip, such as quasi-brittle, elastic-plastic etc. Choice of the suitable critical distance is crucial.

Unfortunately, the convergence of the results with increasing number of the Williams expansion terms is not unambiguous and therefore any specific recommendation on the number of the terms, that shall be considered during the fracture analysis, cannot be given.

The latter analysis deals with estimation of the crack deflection angle on SCB specimens with asymmetric supports during the 3 PB test, see Fig. 1b. Regarding the previous analysis, the following results are presented for critical distance $r_{c}=1 \mathrm{~mm}$. In Fig. 3, results for both relative crack lengths 0.2 and 0.5 are introduced. Altogether eight combinations of various supports distances from the middle of specimens were solved excluding the configurations when the crack closure occurs. Particularly, $S_{2}$ was kept 30 and then $40 \mathrm{~mm}$ and the distance $S_{1}$ was varied from 10 to $40 \mathrm{~mm}$, see the plots in Fig. 3 where again the dependence of the crack deflection angle on the stress intensity factors ratio is plotted. 


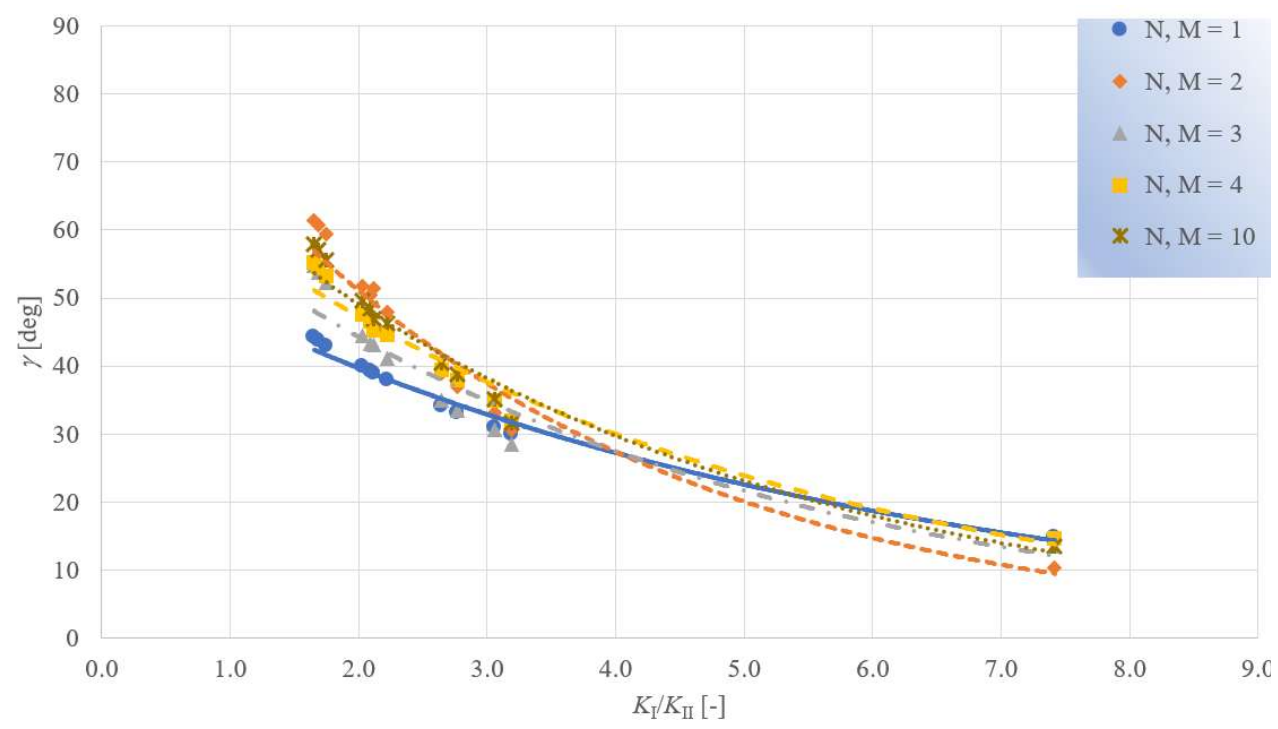

a) $a / R=0.2$ and $S_{2}=30 \mathrm{~mm}$

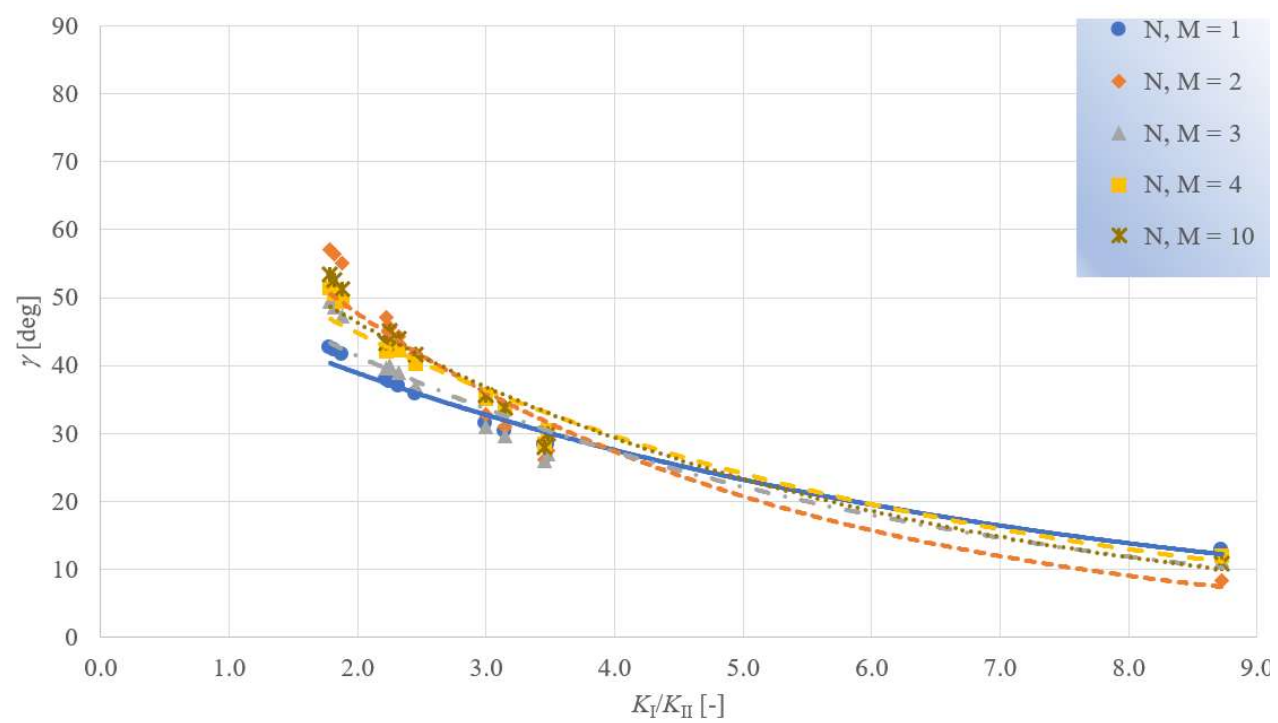

b) $a / R=0.2$ and $S_{2}=40 \mathrm{~mm}$ 


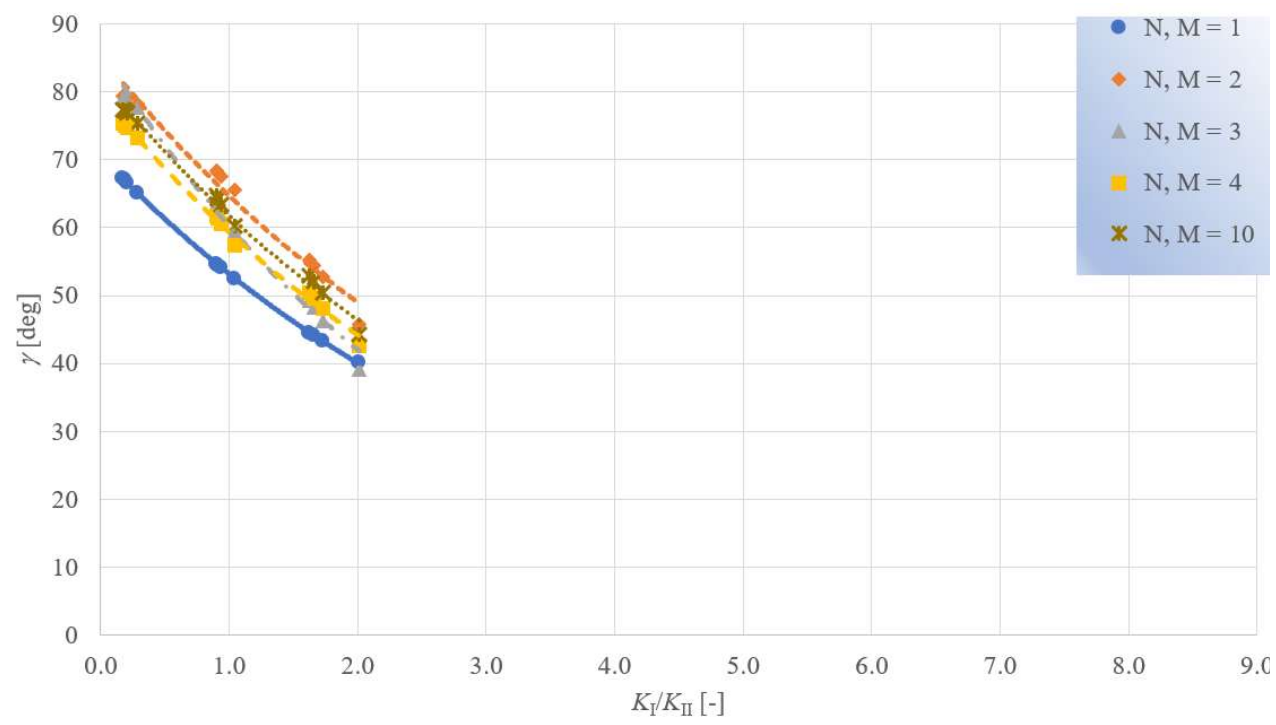

c) $a / R=0.5$ and $S_{2}=30 \mathrm{~mm}$

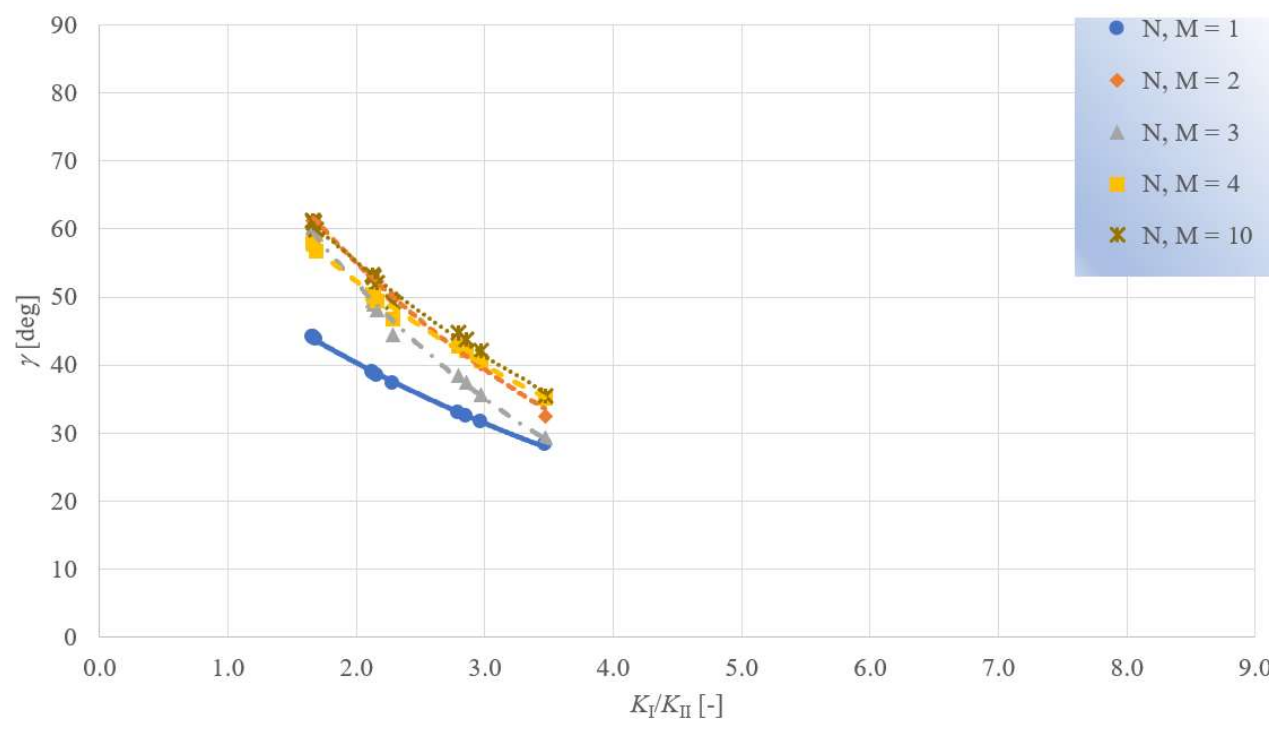

d) $a / R=0.5$ and $S_{2}=40 \mathrm{~mm}$

Fig. 3. Dependences of the crack deflection angle $\gamma$ on the $K_{\mathrm{I}} / K_{\mathrm{II}}$ ratio obtained on SCB specimens with various supports asymmetries at the critical distances $r_{c}=1 \mathrm{~mm} ; N$ and $M$ represents the numbers of the initial terms considered in Williams expansion for $\sigma_{\theta \theta}$ approximation.

Evaluation of the results obtained on SCB configurations with asymmetric supports brings qualitatively the same conclusions. Moreover, it is visible, that when the crack is longer, the stress intensity factors for mode I and II of loading are similar (especially when $S_{2}=30 \mathrm{~mm}$ ), i.e. their ratio is close to one and therefore the differences between the results obtained via classical one-parameter and generalized multi-parameter MTS are quite large for all the configurations investigated. 


\section{Conclusions}

Multi-parameter form of the well-known MTS criterion for estimation of the further crack propagation angle under mixed-mode conditions has been applied on a large range of crack configurations in a SCB specimen. Especially, the effect of the crack eccentricity and supports asymmetry has been investigated. It has been found out that for specific configuration it is more relevant to consider more than only the first singular term in the Williams expansion for the crack-tip stress field approximation. It has been shown that when the stress intensity factors ratio decreases, the importance of the higher-order terms increases. Unfortunately, any specific recommendation on the definite number of the WE terms could not be formulated. A proper choice of the critical distance for application of the fracture criterion has been discussed shortly.

Financial support from the Czech Science Foundation (project No. 18-12289Y) and from the Faculty of Civil Engineering, Brno University of Technology (project No. FAST-S-19-5896) is gratefully acknowledged.

\section{References}

1. F. Berto, P. Lazzarin, Int. J. Fract. 161, 221 (2010)

2. Z.Z. Du, J.W. Hancock, J. Mech. Phys. Solids 39, 555 (1991)

3. B.L. Karihaloo, B.L., Int. J. Fract. 95, 379 (1999)

4. M.L. Williams, J. Appl. Mech. 24, 109 (1957)

5. C. Hou, Z. Wang, W. Liang, J. Li, J., Theor. Appl. Fract. Mech. 85, 355 (2016)

6. D.J. Smith, M.R. Ayatollahi, M.J. Pavier, M. J., Fatigue Fract. Engng Mater. Struct. 24(2), 137 (2001)

7. M.R. Ayatollahi, M. Nejati, Fatigue Fract. Eng. Mater. Struct. 34, 159 (2010)

8. M.R. Ayatollahi, M. Nejati, Int. J. Mech. Sci. 53, 164 (2011)

9. L. Malíková, Engng. Fract. Mech. 143, pp. 32 (2015)

10. L. Malíková, V. Veselý, Fatigue Fract. Engng Mater. Struct. 38, 91 (2014)

11. L. Malíková, V. Veselý, Theor. Appl. Fract. Mech. 91, 25 (2017)

12. V. Veselý, P. Frantík, J. Sobek, L. Malíková (Šestáková), S. Seitl, Fatigue Fract. Engng. Mater. Struct. 7(25), 69 (2014)

13. B.L. Karihaloo, Q.Z. Xiao, Engng. Fract. Mech. 68(15), 1609 (2001)

14. P. Tong, T.H.H. Pian, S.J. Lasry, Int. J. Numer. Methods Eng. 7(3), 297 (1973)

15. Q.Z. Xiao, B.L. Karihaloo, X.Y. Liu, Int. J. Fract. 125(3), 207 (2004)

16. L. Šestáková, Appl. Mech. Mater. 245, 120 (2013)

17. F. Erdogan, G.C. Sih, J. Basic Engng. 85, 519 (1963)

18. A. Seweryn, A. Lukaszewicz, Fract. Mech. 69, 1487 (2002)

19. G.C. Sih, J.W. Ho, J. Theor. Appl. Fract. Mech. 16, 179 (1991)

20. L. Susmel, D. Taylor, Engng. Fract. Mech. 75, 534 (2008)

21. ANSYS Program Documentation. User's manual version 19.0. Swanson Analysis System, Inc., Houston (2019) 